\title{
Insights from Monitoring Aspirin Adherence: A Medication Adherence Cascade Tool
}

\author{
Heather L Wheat' \\ Elliane Irani ${ }^{2}$ \\ Joel Hughes ${ }^{3}$ \\ Richard Josephson ${ }^{4,5}$ \\ Mary A Dolansky ${ }^{2}$ \\ 'Department of Medicine, Case Western \\ Reserve University, University Hospitals \\ Cleveland Medical Center, Cleveland, \\ $\mathrm{OH}$, USA; ${ }^{2}$ Frances Payne Bolton School \\ of Nursing, Case Western Reserve \\ University, Cleveland, OH, USA; \\ ${ }^{3}$ Department of Psychological Sciences, \\ Kent State University, Kent, OH, USA; \\ ${ }^{4}$ School of Medicine, Case Western \\ Reserve University, Cleveland, OH, USA; \\ ${ }^{5}$ Department of Medicine, Division of \\ Cardiology, Case Western Reserve \\ University, Harrington Heart \& Vascular \\ Institute, University Hospitals Cleveland \\ Medical Center, Cleveland, OH, USA
}

Background: Adherence to recommended medications is a key issue in the care of patients with cardiovascular disease (CVD) and barriers to adherence are well established during the medication adherence cascade, the processes of prescribing, obtaining, taking, and maintaining medication use. Aspirin avoids many of the barriers in the medication adherence cascade as it does not require a prescription (prescribing) and is inexpensive, easily accessible (obtaining), prescribed once-daily (taking) as an over-the-counter medication and is generally perceived by patients as safe (maintaining). The purpose of this paper is to report aspirin adherence and propose the Medication Adherence Cascade Tool to assist clinicians to consider all aspects of medication adherence.

Methods: Adherence to aspirin was monitored with an electronic pillbox. Frequency analysis, independent $T$-tests, and ANOVA were completed on 151 patients with underlying heart failure who were prescribed aspirin within a larger parent study. Chi-square tests were completed to assess differences in baseline demographic characteristics.

Findings: Mean aspirin adherence was $82.2 \%$ overall, with $11.9 \%$ of sample with adherence $\leq 50 \%, 18.5 \%$ with adherence $50-80 \%$, and $69.5 \%$ with adherence $\geq 80 \%$. Greater adherence was observed in self-identified White as compared to Black patients $(84.47 \%$ vs $73.53 \% ; \mathrm{p}=0.014)$, and patients $\geq 70$ years of age compared to $<70$ years $(87.00 \%$ vs $77.49 \% ; \mathrm{p}=0.009)$

Interpretation: Aspirin adherence was suboptimal despite the fact that it addresses most of the barriers on the medication adherence cascade (ie, relatively easy access, low cost, and low risk). A Medication Adherence Cascade Tool (MACT) is proposed as a clinical guide to facilitate patient-provider co-production of strategies to address medication adherence. The tool can assist patients and providers to co-produce adherence to achieve optimal medication benefits.

Keywords: medication adherence, aspirin, cardiovascular disease

\section{Introduction}

Patients with cardiovascular disease (CVD) take several medications with proven mortality benefit, in addition to numerous other medications for associated comorbidities. Taking medications is essential to realize the evidence-based benefits. ${ }^{1}$ Many system and personal factors contribute to overall medication adherence, with adherence being a multi-step, complex process. ${ }^{2}$ Patients may choose to ration medications due to cost, spread out doses to minimize side effects, or choose not to take medications under certain circumstances, such as travel. ${ }^{3}$ Many factors are linked to poorer medication adherence, including cost of medications, perceived or burdensome side effects, polypharmacy, health literacy, complexity of the medication regimen, and lack
Correspondence: Heather L Wheat Department of Medicine, Case Western Reserve University, University Hospitals Cleveland Medical Center, II 100 Euclid Ave, Cleveland, $\mathrm{OH}, 44106$, USA Tel +I 216844-8447

Email Heather.Wheat@UHhospitals.org 
of social support. ${ }^{2,4-9}$ Similarly, younger age and certain comorbidities, including depression and underlying cognitive impairment are associated with decreased medication adherence. ${ }^{10-12}$ Aspirin is an ideal medication to understand medication adherence in chronic disease, like CVD as it is taken just once daily, has relatively limited side effects, and is perceived as one of the safest medications. ${ }^{13,14}$ Almost 30 million Americans over the age of 40 years take aspirin, including $>50 \%$ of those over 70 years, whether the medication is prescribed or not, in order to prevent CVD. ${ }^{15}$ Aspirin is easily accessible and inexpensive, can be purchased over the counter, and is widely prescribed for both primary and secondary prevention for patients at risk of or with underlying CVD. Thus, the study of aspirin adherence is ideal as it eliminates the common barriers identified in the literature and are listed in our medication adherence cascade that includes the steps of prescribing, obtaining, taking and maintaining medications (Figure 1).

The purpose of this paper was to 1) report the rate of adherence to aspirin use in a sample of adults with CVD, 2) identify differences in adherence rates based on demographic factors, and 3) propose adoption of the Medication Adherence Cascade Tool (MACT) to improve medication adherence within the context of CVD. Our study addresses many of these aforementioned barriers to medication adherence and provides contemporary data in the setting of an ideal drug for study. This analysis sheds light on what healthcare providers can expect for medication adherence in the most pragmatic of circumstances and offers a useful clinical tool to guide intervention to improve medication adherence in chronic disease.

\section{Materials and Methods}

This is a secondary analysis of data from the Heart Adherence, Behavior, and Cognition (HeartABC) study, which was a longitudinal, observational study of 372 community-dwelling adult patients with CVD, specifically heart failure (HF), that focused on the psychosocial and cognitive factors that impact HF self-management. ${ }^{10}$ The current analysis was performed on the 151 patients with underlying HF who were prescribed aspirin for either primary or secondary prevention of atherosclerotic disease (myocardial infarction, peripheral vascular disease), by their healthcare provider from various cardiology practices within two major hospital systems in Northeast Ohio. Participant eligibility for the parent study included a diagnosis of systolic HF (with confirmation of diagnosis with left ventricular ejection fraction $<40 \%$ for at least 3 months and within the 36 months prior to study enrollment), age 50-85 years at the time of enrollment, and classification as NYHA class II or III for $\geq 3$ months by their physician. Individuals were ineligible if they had

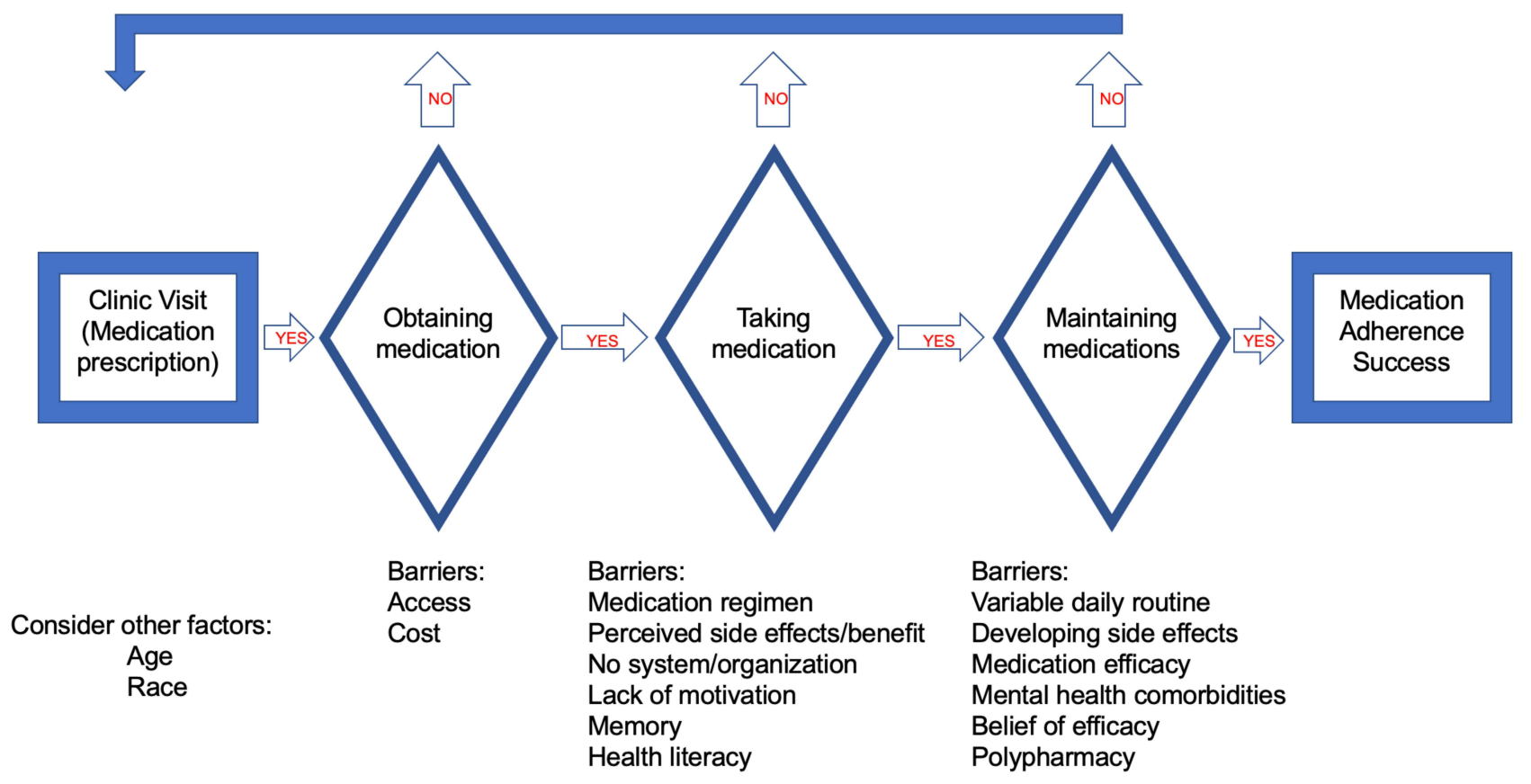

Figure I Author-generated clinical tool for medication adherence that outlines key steps that ultimately lead to medication adherence. There are several barriers at each step that can lead to non-adherence. The Medication Adherence Cascade Tool is suggested as a strategy to facilitate patient-provider co-production of medication adherence. 
cardiac surgery within 3 months prior to enrollment, were using a home telehealth HF monitoring program, or had overt cognitive dysfunction. Cognitive dysfunction included a history of neurological disorder or injury (eg, Alzheimer's disease, dementia, stroke, seizures), moderate or severe head injury, past or current significant psychiatric disorders (eg, psychotic disorders, bipolar disorder, learning disorder, development disability), renal failure requiring dialysis, untreated sleep apnea, and substance abuse currently or within the past 5 years.

All patients were recruited from either inpatient cardiology services or outpatient cardiology practices in Northeast Ohio and provided written informed consent for their participation. The majority of patients were on at least four medications for prescription of guidelinedirected medical therapy for their underlying HF. Most patients were insured through Medicare or Medicaid. All procedures and use of human test subjects were approved by the Institutional Review Boards of Kent State University, Summa Health System, and University Hospitals Cleveland Medical Center (STUDY20180810 renewal 7/2020). The study was conducted in accordance with the Declaration of Helsinki. Permission was obtained from the owners of the original dataset (Drs Hughes and Dolansky) to use the information in the database for the purposes of this research and data analysis, and all patient information was de-identified. After recruitment and written consent, a research assistant obtained baseline demographics and clinical data from official medical records and completed a series of self-report questionnaires including neuropsychological testing (Visit 1). Approximately 2 weeks later at visit 2, a research assistant installed and filled an electronic pillbox in each patient's home and instructed the patient on its use, in order to collect adherence data for 21 days. Baseline characteristics on depression were obtained using the Patient Health Questionnaire (PHQ-9), a validated calculator that uses patient answers to nine questions in order to identify underlying depression. A score of 5 points suggests mild depression, which was included in this study as the cut-off for inclusion of baseline depression.

Medication adherence was measured objectively using MedSignals ${ }^{\circledR}$ Pillbox (VitalSignals, LLC, Lexington, KY). MedSignals pillbox was selected based on ease of participant use, ability to monitor multiple medications simultaneously, and the capacity to transmit daily adherence data via Bluetooth and home phone to a secure electronic server. Research assistants were readily available to participants, were trained on installation, problem solving, and instructing participants on the use of the pillbox, and provided technical support to patients during the study. The pillbox tracked adherence data for up to four patient medications for 21 days. All patients were counseled on their medical regimen. Adherence was defined as the percent of days that the patient was compliant with their personally prescribed medication regimen divided by the number of total days monitored (possible range of scores $=0-100 \%)$. Although the MedSignals device had a variety of audible and alarm features, all were deactivated during the study period.

Frequency analyses were completed to assess aspirin adherence rates. Despite a left-skewed distribution for medication adherence, normality was assumed due to a large sample size. Similarly, Levene's tests for all analyses were performed with assumption of equal variances. Independent sample $T$-tests and ANOVA were performed to assess aspirin adherence related to baseline characteristics. Chi-square tests were performed to assess differences in baseline characteristics in patients with adherence $\leq 50 \%, 50-80 \%$ and $\geq 80 \%$. Post hoc Bonferroni adjustments were used to further assess significant values. A p-value of $<0.05$ was considered statistically significant for all analyses. Comparison of aspirin with other common HF medications was not performed as the purpose of the paper was to understand aspirin adherence as it addresses most of the factor related to adherence in the literature. All analyses were performed using IBMC Statistical Package for the Social Sciences (SPSSC) version 26.0 statistical software (IBM Corporation).

The Medication Adherence Cascade Tool was developed by the authors after drawing from existing CVD literature on factors that affect medication adherence (ie prescribing, obtaining, taking, and maintaining). An expert in cardiovascular care and self-management assisted in the development and review of the tool.

\section{Results}

The demographics of the sample and description of aspirin use are reported in Table 1. Of the 151 aspirin users, the average age was $68.9 \pm 10.05$ years, $57.6 \%$ were male, and $78.8 \%$ self-identified as White. Overall average adherence to aspirin was $82.2 \%$ (SD 22.4). The majority of the sample, $69.5 \%$, met a standard definition of medication adherence ( $\geq 80 \%$ adherent), and $30.5 \%$ had perfect adherence (100\%; Figure 2). ${ }^{16}$ Other adherence cutoffs were as follows: $11.9 \%$ of the patients had $\leq 50 \%$ adherence, 
Table I Baseline Patient Characteristics

\begin{tabular}{|c|c|c|c|c|}
\hline Characteristic & & $\begin{array}{c}\text { Total Sample } \\
\text { N = I5 I } \\
\text { n (\%) }\end{array}$ & $\begin{array}{c}\text { Mean \% Aspirin } \\
\text { Adherence } \\
\text { Mean (standard error) }\end{array}$ & p-value \\
\hline \multirow[t]{2}{*}{ Gender } & Male & $87(57 \cdot 6)$ & $83 \cdot 11(2 \cdot 372)$ & \\
\hline & Female & $64(42 \cdot 4)$ & $80.84(2.857)$ & 0.540 \\
\hline \multirow[t]{2}{*}{ Race } & White & $119(78.8)$ & 84.47 ( I .985) & \\
\hline & Black & $32(2 \mid \cdot 2)$ & $73 \cdot 53(4 \cdot 120)$ & $0.014 * *$ \\
\hline Age & $\begin{array}{l}70 \text { years } \\
<70 \text { years }\end{array}$ & $\begin{array}{l}74(49.0) \\
77(5 \mid 0)\end{array}$ & $\begin{array}{l}87.00(2.509) \\
77.49(2.54 I)\end{array}$ & $0.009 * *$ \\
\hline \multirow[t]{2}{*}{ Depression* } & Yes & $67(44 \cdot 4)$ & $80.33(2.630)$ & \\
\hline & No & $84(55 \cdot 6)$ & $83.6 I(2.54 I)$ & 0.373 \\
\hline \multirow[t]{2}{*}{ Prior MI } & Yes & $81(53 \cdot 6)$ & $81 \cdot 77(2 \cdot 616)$ & \\
\hline & No & $70(46 \cdot 4)$ & $82 \cdot 60(2 \cdot 526)$ & 0.820 \\
\hline PVD & $\begin{array}{l}\text { Yes } \\
\text { No }\end{array}$ & $\begin{array}{l}20(13 \cdot 2) \\
|3|(86 \cdot 8)\end{array}$ & $\begin{array}{l}81.90(5.666) \\
82 \cdot 19(1.923)\end{array}$ & 0.957 \\
\hline \multirow[t]{4}{*}{ Marital status } & Never married & $7(4 \cdot 6)$ & $73 \cdot 43(8 \cdot 560)$ & \\
\hline & Married & $82(54 \cdot 3)$ & $84 \cdot 85(2 \cdot 25 \mathrm{I})$ & \\
\hline & Widowed & $27(17 \cdot 9)$ & $86 \cdot 37(3.993)$ & \\
\hline & $\begin{array}{l}\text { Separated } \\
\text { Divorced }\end{array}$ & $\begin{array}{c}5(3 \cdot 3) \\
30(19 \cdot 9)\end{array}$ & $\begin{array}{c}77.80(10.716) \\
73.73(4.930)\end{array}$ & $0 \cdot 104$ \\
\hline \multirow[t]{4}{*}{ Education } & High school or less & $61(40 \cdot 4)$ & $82 \cdot 41(2 \cdot 684)$ & \\
\hline & Technical/trade school & $15(9 \cdot 9)$ & $90 \cdot 13(3.052)$ & \\
\hline & Some college & $44(29 \cdot 1)$ & $78 \cdot 41(4 \cdot 164)$ & \\
\hline & Bachelor or master degree & $31(20 \cdot 5)$ & $83 \cdot 10(3 \cdot 660)$ & 0.365 \\
\hline \multirow[t]{3}{*}{ Employment } & Retired & $101(66 \cdot 9)$ & $82 \cdot 86(2 \cdot 284)$ & \\
\hline & Part/full time & $42(27 \cdot 8)$ & $81 \cdot 62(3 \cdot 357)$ & \\
\hline & Homemaker & $8(5 \cdot 3)$ & $76.00(7.008)$ & 0.697 \\
\hline
\end{tabular}

Notes: *Depression was calculated using the Patient Health Questionnaire (PHQ-9) standardized cut-off of 5 points. **Statistically significant using $\mathrm{P}$-value $<0.05$. Abbreviations: MI, myocardial infarction; PVD, peripheral vascular disease.

$18.5 \%$ of the patients had adherence between $50 \%$ and $80 \%$, and $69.5 \%$ of the patients had adherence $\geq 80 \%$.

Independent sample $t$-testing demonstrated that Whites as compared with Blacks (mean adherence $84.47 \%$ versus $73.53 \%, p=0.014$ ), and age $\geq 70$ years compared to younger participants (mean adherence $87.00 \%$ versus $77.49 \%, p=0.009$ ) had statistically significant higher adherence rates (Table 1). There were no statistically significant differences in aspirin adherence based on gender, a history of myocardial infarction, peripheral vascular disease, or depression, marital status, education level, or employment. When comparing mean aspirin adherence among all baseline factors, patients who attended technical/trade school had the highest overall adherence (Figure 3). Of those meeting the standard cut-off of $80 \%$ adherence compared to those with $<80 \%$ adherence, baseline characteristics were statistically different with regard to race and age, as above. When comparing patients with $\leq 50 \%$ adherence to those with $50-80 \%$, and $\geq 80 \%$ adherence, of those who had $\geq 80 \%$ adherence, patients $\geq 70$ years had statistically significant greater adherence than to those $<70$ years old $(p=0.0069)$. 


\section{Aspirin Adherence}

60

50

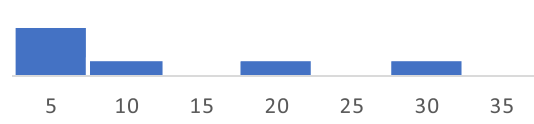

Figure 2 Overall distribution of sample aspirin adherence.

\section{Discussion}

This study focused on aspirin as an ideal case to understand medication adherence and to offer a benchmark for what providers can expect for medication adherence when prescribing a drug regimen in patients with CVD. Aspirin is an ideal medication for defining adherence due to its ease of access, once-daily use, inexpensive cost, limited side effects, and relative widespread national use. However, even when such defining factors are present, this study demonstrated that medication adherence remains suboptimal. This is important to consider in a sample of patients who voluntarily enrolled in a research study, physically had aspirin in a monitored pillbox, and were educated about their chronic disease and importance of taking medications.

Rieckmann and colleagues previously reported on aspirin adherence following an acute coronary event in a population with depression using an electronic pill bottle cap to monitor adherence. ${ }^{17}$ In the Rieckmann analysis, the average aspirin adherence among patients ranged from $76.1 \%$ to $89.5 \%$, depending on underlying depression status. This study was important as aspirin is a crucial medication following an acute coronary event. However, the Rieckmann analysis has important differences. For example, their study focuses on medication adherence in the acute setting and used a single cut-off value (75\%) for adherence. Our study expands upon understanding medication adherence in several important ways. First of all, while the Rieckmann study focused on an acute event, most patients taking prescribed medications will require lifelong treatment to achieve benefit, especially in the setting of chronic disease, such as HF. Furthermore, in the setting of underlying comorbidities, most patients are prescribed more than just one medication. While our analysis focused on aspirin adherence as a benchmark, up to four medications could be placed in the electronic pillboxes for monitoring. Hawthorne effect therefore must be considered in the Rieckmann analysis, as patients are more likely to take a single medication with limited side effects, 


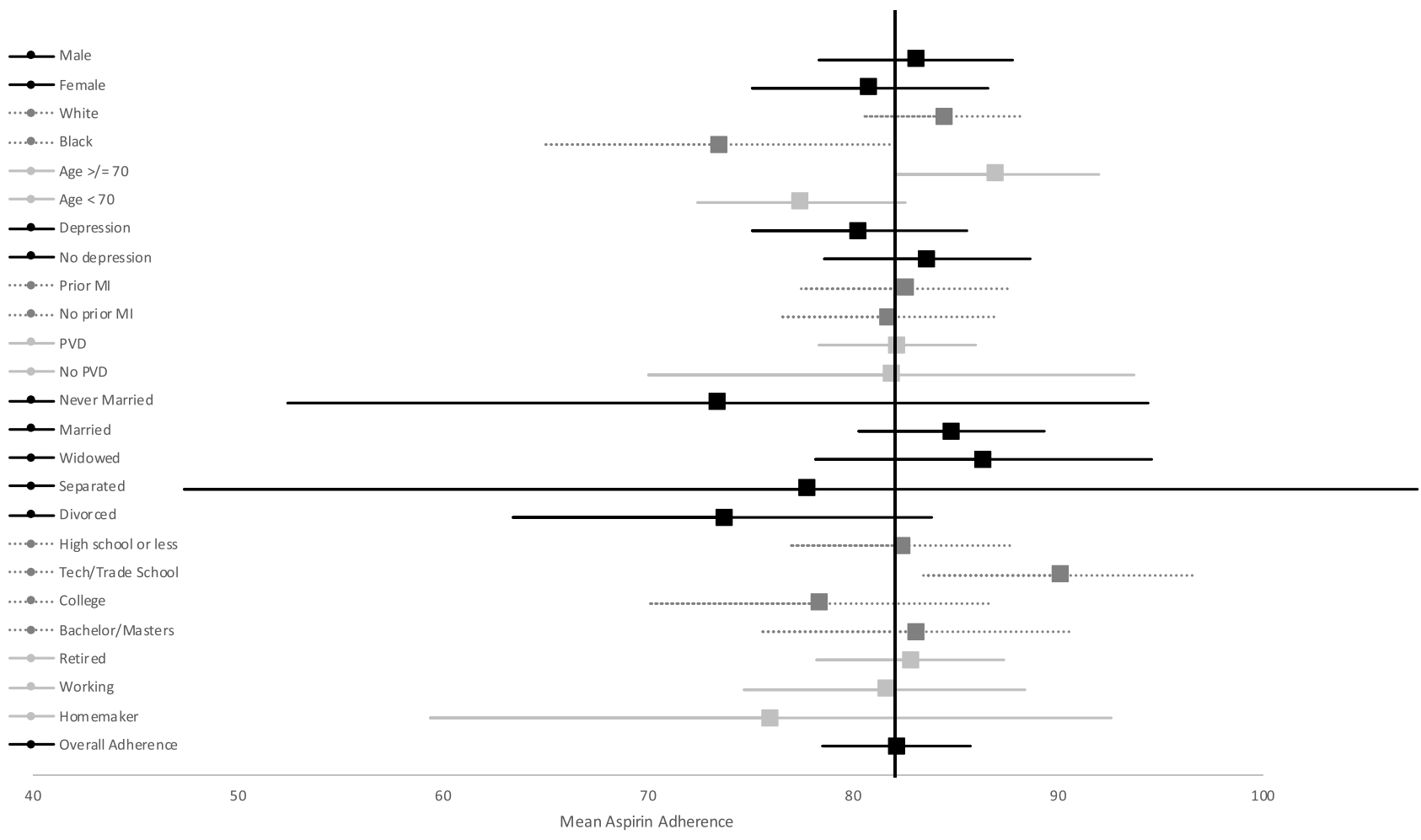

Figure 3 Forest plot demonstrating medication adherence by baseline characteristics as compared to the overall mean sample aspirin adherence (vertical line).

especially following an acute, life-altering event, such as acute coronary syndrome. Similarly, our study included community-dwelling patients living with a chronic disease, excluding patients living in a nursing facility or with access to home care or a visiting nursing program, which the Rieckmann study did not.

Another important aspect of our study is defining a distribution of adherence amongst patients prescribed aspirin (Figure 2). While the Rieckmann study focused on a single cut-off value for medication adherence $(75 \%)$, our study helps demonstrate the distribution of adherence among patients with a chronic disease. ${ }^{17}$ Among the patients meeting a standard medication adherence of $>80 \%$, we did identify that White patients and patients $\geq 70$ years of age were more compliant than Black patients and those $<70$ years. ${ }^{16}$ However, our adherence distribution demonstrates that despite several barriers (Figure 1) to medication adherence including access, cost, and education of their medication regimen, having been eliminated with use of aspirin as a benchmark drug with minimal side effects, healthcare providers can still expect reduced medication adherence. Furthermore, practitioners can anticipate worse adherence rates when considering more complex medication regimens or difficult dosing schedules in medications with more side effects.

Understanding the distribution of adherence is important for healthcare providers prescribing medications to patients with chronic diseases. While prior studies have detailed adherence rates in such patients, most record adherence based on pharmacy records or medication lists. ${ }^{4,7,18}$ For example, in a study by Dunlay and colleagues, medication adherence among community-dwelling patients with $\mathrm{HF}$ was determined using pharmacy refill records. ${ }^{4}$ However, refill history does not provide information on whether or not patients actually took the medication once it was in their possession. Therefore, use of the electronic pillbox in our study provides a more accurate representation of medication adherence over a given period of time.

Of note, no significant difference was demonstrated in patients taking aspirin for primary or secondary prevention (patients with prior myocardial infarction or underlying peripheral vascular disease; patients with a prior cerebrovascular event were excluded). This may be related to the perceived importance and relative safety of aspirin, as well as the relative 
ease of administration (one pill, once-a-day). This is further supported by the more than 6 million Americans who take a daily aspirin without the prescription from a physician. ${ }^{15}$ Aspirin adherence in secondary prevention in patients who have a history of myocardial infarction or stroke has been extensively studied, but we were unable to find comparisons of adherence between primary and secondary prevention.

The clinical implications of poor medication adherence are not inconsequential. Poor adherence is associated with increased hospitalizations, increased healthcare costs, and mortality. ${ }^{1,11,19}$ Many systematic reviews are available that identify factors associated with medication adherence in order to better understand and improve medication adherence to avoid such adverse events. ${ }^{5,13,20-23}$. Yet, there are few clinical tools that include these factors to assist physicians and interprofessional teams to address potential issues with medication adherence. The Medication Adherence Cascade Tool (Figure 1) was developed from common barriers published in the literature and is proposed to assist healthcare providers to work collaboratively with patients to identify barriers and potential strategies to address these barriers.

The Medication Adherence Cascade Tool was developed to highlight the steps in medication adherence that involves prescribing, obtaining, taking and maintaining medications and highlights barriers at each step. The tool provides a framework to help providers and patients to understand the steps in medication adherence and opportunity to identify barriers in each of the steps. The tool supports patients and healthcare providers to co-produce an individual approach to target adherence strategies. Adherence strategies can be different for each step of the cascade. Healthcare providers and patients start at the left side of the tool and work collaboratively over time through each step to identify barriers and implement strategies for each step. Strategies might include education, automatic prescription refills, use of reminder systems, such as electronic bill bottles or alarms, and treating underlying mental health conditions. ${ }^{13}$

The Medication Adherence Cascade Tool outlines the key steps that ultimately lead to successful medication adherence and the potential barriers at each step. The healthcare provider and patient use the tool together to identify barriers in each of the steps and agree upon strategies to address the barriers. This co-production of barrier awareness and identification of strategies occurs over time as non-adherence is a dynamic process that typically is related to many barriers.

Potential limitations to this study include that the 21-day monitoring period was relatively short for a medication that is often taken for long periods of time. However, this duration is similar to other medication adherence studies with monitoring durations from 30 to 90 days. ${ }^{17,20,21}$ Furthermore, observation of suboptimal medication adherence within this short duration in research subjects who knew that they were being monitored provides an even greater indication that such suboptimal medication adherence can be expected in the real-world setting. Patients may have had difficulty with utilization of the electronic pillbox. However, each patient was individually instructed in their home regarding pillbox use, and trained research assistants were readily available to address perceived and real difficulties. This study also excluded patients with underlying cognitive impairment, psychiatric disorder, and substance abuse, among other exclusion criteria, and therefore may not be representative of the general population. However, patients with such conditions as listed above often have worse adherence to medications, and thus would likely further demonstrate poorer rates of adherence overall. ${ }^{10,24-26}$ The Medication Adherence Cascade Tool (Figure 1) was used as a framework that was developed in response to our findings and was not tested. Future research is needed to assess the usability, acceptability, and feasibility of the tool with external validation.

\section{Conclusions}

This study, by focusing on the monitored adherence of oncedaily aspirin, a medication that was in the patients' possession, is inexpensive, and is generally perceived as safe, provides novel and important insights into medication adherence in those with CVD. Even when many of the well-described factors adversely influencing medication adherence are minimized or circumvented, adherence is suboptimal in many (as much as $30 \%$ ) of adult patients. The Medication Adherence Cascade Tool assists clinicians and patients with the identification of barriers to adherence and development of a plan that addresses these barriers. Use of aspirin as a benchmark drug in such pragmatic settings illustrates what healthcare providers can expect in terms of adherence to the medications that they prescribe and provides support for optimal care using a clinical tool such as the MACT.

\section{Funding}

The parent study was funded by the National Heart, Lung, and Blood Institute (R01HL096710-01A1 awarded to Drs Hughes and Dolansky).

\section{Disclosure}

The authors report no conflicts of interest in this work. 


\section{References}

1. Ruppar TM, Cooper PS, Mehr DR, Delgado JM, Dunbar-Jacob JM. Medication adherence interventions improve heart failure mortality and readmission rates: systematic review and meta-analysis of controlled trials. J Am Heart Assoc. 2016;5(6). doi:10.1161/ jaha.115.002606

2. Wu J-R, Moser DK, Lennie TA, Burkhart PV. Medication adherence in patients who have heart failure: a review of the literature. Nurs Clin North Am. 2008;43(1):133-153. doi:10.1016/j.cnur.2007.10.006

3. Piette JD, Heisler M, Wagner TH. Cost-related medication underuse among chronically III adults: the treatments people forgo, how often, and who is at risk. Am J Public Health. 2004;94(10):1782-1787. doi:10.2105/ajph.94.10.1782

4. Dunlay SM, Eveleth JM, Shah ND, Mcnallan SM, Roger VL. Medication adherence among community-dwelling patients with heart failure. Mayo Clin Proc. 2011;86(4):273-281. doi:10.4065/ mcp.2010.0732

5. Gellad WF, Grenard JL, Marcum ZA. A systematic review of barriers to medication adherence in the elderly: looking beyond cost and regimen complexity. Am J Geriatr Pharmacother. 2011;9(1):11-23. doi:10.1016/j.amjopharm.2011.02.004

6. Koh JJK, Cheng RX, Yap Y, et al. Access and adherence to medications for the primary and secondary prevention of atherosclerotic cardiovascular disease in Singapore: a qualitative study. Patient Prefer Adherence. 2018;12:2481-2498. doi:10.2147/ppa.s176256

7. Kulkarni SP, Alexander KP, Lytle B, Heiss G, Peterson ED. Longterm adherence with cardiovascular drug regimens. Am Heart $J$. 2006;151(1):185-191. doi:10.1016/j.ahj.2005.02.038

8. Osborn CY, Kripalani S, Goggins KM, Wallston KA. Financial strain is associated with medication nonadherence and worse self-rated health among cardiovascular patients. $J$ Health Care Poor Underserved. 2017;28(1):499-513. doi:10.1353/hpu.2017.0036

9. Wu J-R, Lennie TA, Chung ML, et al. Medication adherence mediates the relationship between marital status and cardiac event-free survival in patients with heart failure. Heart Lung. 2012;41 (2):107-114. doi:10.1016/j.hrtlng.2011.09.009

10. Dolansky MA, Hawkins MA, Schaefer JT, et al. Association between poorer cognitive function and reduced objectively monitored medication adherence in patients with heart failure. Circ Heart Fail. 2016;9 (12). doi:10.1161/circheartfailure.116.002475

11. Gathright EC, Dolansky MA, Gunstad J, et al. The impact of medication nonadherence on the relationship between mortality risk and depression in heart failure. Health Psychol. 2017;36(9):839-847. doi: $10.1037 /$ hea0000529

12. Hawkins LA, Kilian S, Firek A, Kashner TM, Firek CJ, Silvet H. Cognitive impairment and medication adherence in outpatients with heart failure. Heart Lung. 2012;41(6):572-582. doi:10.1016/j. hrtlng.2012.06.001

13. Bosworth HB, Granger BB, Mendys P, et al. Medication adherence: a call for action. Am Heart J. 2011;162(3):412-424. doi:10.1016/j. ahj.2011.06.007

Patient Preference and Adherence

\section{Publish your work in this journal}

Patient Preference and Adherence is an international, peer-reviewed, open access journal that focusing on the growing importance of patient preference and adherence throughout the therapeutic continuum. Patient satisfaction, acceptability, quality of life, compliance, persistence and their role in developing new therapeutic modalities and compounds to optimize clinical outcomes for existing disease
14. Cullen G, Kelly E, Murray FE. Patients' knowledge of adverse reactions to current medications. Br J Clin Pharmacol. 2006;62 (2):232-236. doi:10.1111/j.1365-2125.2006.02642.x

15. Widespread aspirin use despite few benefits, high risks. ScienceDaily; July 22, 2019. Available from: https://www.scienceda ily.com/releases/2019/07/190722182126.htm. Accessed July 28, 2020.

16. Monane M. The effects of initial drug choice and comorbidity on antihypertensive therapy compliance results from a population-based study in the elderly. Am $J$ Hypertens. 1997;10(7):697-704. doi:10.1016/s0895-7061(97)00056-3

17. Rieckmann N, Kronish IM, Haas D, et al. Persistent depressive symptoms lower aspirin adherence after acute coronary syndromes. Am Heart J. 2006;152(5):922-927. doi:10.1016/j.ahj.2006.05.014

18. Naderi SH, Bestwick JP, Wald DS. Adherence to drugs that prevent cardiovascular disease: meta-analysis on 376,162 patients. Am J Med. 2012;125(9):882-887. doi:10.1016/j.amjmed.2011.12.013

19. Bitton A, Choudhry NK, Matlin OS, Swanton K, Shrank WH. The impact of medication adherence on coronary artery disease costs and outcomes: a systematic review. Am J Med. 2013;126(4):357.e7-357. e27. doi:10.1016/j.amjmed.2012.09.004

20. Schwartz JK. Pillbox use, satisfaction, and effectiveness among persons with chronic health conditions. Assist Technol. 2016;29 (4):181-187. doi:10.1080/10400435.2016.1219884

21. Arnet I, Rothen J-P, Albert V, Hersberger K. Validation of a novel electronic device for medication adherence monitoring of ambulatory patients. Pharmacy. 2019;7(4):155. doi:10.3390/pharma cy7040155

22. Murray MD, Young J, Hoke S, et al. Pharmacist intervention to improve medication adherence in heart failure. Ann Intern Med. 2007;146(10):714. doi:10.7326/0003-4819-146-10-20070515000005

23. Peterson AM, Takiya L, Finley R. Meta-analysis of trials of interventions to improve medication adherence. Am J Health Syst Pharm. 2003;60(7):657-665. doi:10.1093/ajhp/60.7.657

24. Cho MH, Shin DW, Chang S-A, et al. Association between cognitive impairment and poor antihypertensive medication adherence in elderly hypertensive patients without dementia. Sci Rep. 2018;8(1). doi:10.1038/s41598-018-29974-7

25. Gathright EC, Dolansky MA, Gunstad J, Josephson RA, Moore SM, Hughes JW. Examination of attention, executive function, and memory as predictors of mortality risk in adults with systolic heart failure. Eur J Cardiovasc Nurs. 2019;18(8):729-735. doi:10.1177/ 1474515119863182

26. Stentzel U, van den Berg N, Schulze LN, et al. Predictors of medication adherence among patients with severe psychiatric disorders: findings from the baseline assessment of a randomized controlled trial (Tecla). BMC Psychiatry. 2018;18(1). doi:10.1186/s12888-018$1737-4$ states are major areas of interest for the journal. This journal has been accepted for indexing on PubMed Central. The manuscript management system is completely online and includes a very quick and fair peer-review system, which is all easy to use. Visit http:// www.dovepress.com/testimonials.php to read real quotes from published authors.

\section{Dovepress}

